\title{
NOTE ON A REFLEX MECHANISM IN THE FIXATION OF THE EYEBALLS.
}

\author{
BY W. R. GOWERS, M.D.,

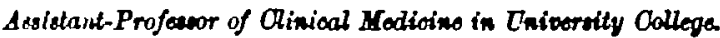

THE phenomens of the fixation of the eyes by which, without conscious effort, they are kept directed to a given point, is attributed to a complex co-ordinating mechanism by which their movements are adjusted. The conditions of this fixation, however, suggest very strongly that its mechanism is not merely under the direction of a volitional centre, but that it is in part reflex. The motor impulses are constantly related to a sensory impression-that proceeding from the "fixing point " of the retina, -and analogy suggests that a reflex relstion between this sensory impulse and the motor impulse may play an important part in the maintenance of the fixation. I am not aware that any recorded facts in physiology or pathology can be regarded as demonstrating the occurrence of such reflex action. The following obserration, however, seems to constitute a proof of its existence.

A man who was lately under my care at the National Hospital for the Paralysed and Epileptic, on account of progressive muscular atrophy, with much rigidity, presented, during the last week of his life, the following symptom:There was apparently a remarkable slowness in the movements of the eyes. If he was told to look at an object at a distance from that at which he was looking (of say $45^{\circ}$ ), his eyes were kept for a moment fixed upon the object he had been looking at, and were then slowly moved until they were fixed on the desired point. On closer examination, however, it was seen 
that this was not merely a slowness of muscular movement. The head was instantly turned towards the object he was told to look at, while the eyes were kept fixed on the first object, and they slowly followed the movement of the head, until they were again in mid-position and fixed on the required point.

It is evident that the maintenance of the fixation of the eyes, while the head was quickly moved, involved a movement of the eyeballa as rapid and considerable as the movement of the head, but in the opposite direction, so as to maintain the fixation of the globes. This movement was evidently independent of, and eren opposed to, the will, and opposed to the voluntary movement of the head, with which that of the eyes is usually associnted. Its source cannot therefore be found in an influence originating in the centres. It must be ascribed to an afferent impression, and the only afferent impression concerned was that visual impression originating from the fixing-point of the retina. The phenomenon seems thus to demonstrate the existence of a reflex mechanism maintaining the fixation of the eyeballs, and capable of doing so during movements of the head. Usually, this mechanism is in such strict subordination to the will, that its existence, apart from the voluntary control, cannot be recognised. In this case it was dissociated from the voluntary action by a resistance to the voluntary control of the reflex centre, and its existence was thus manifested.

It is evident that such a reflex action must be of the greatest importance in maintaining the fixation of the eyeballs during movements of the head, with the least possible expenditure of voluntary action. Probably the mechanism for this reflex is made functionally active during infancy by repeated visual impressions, repeated waves of impulse along the afferent tract. In the young infant no fixation of the eyeballs is to be observed, but after repested visual impressions have occurred for several weeks, an intense impression leads to a reflex fixation; the child's eyes follow a light. The seat of the reflex process is probably the corpora quadrigemina.

The analogy between this reflex inertia of the eyeballs, as it may be termed, and the muscular fixation or rigidity in the limbs, which was very conspicuous in the same patient, is 
obvious. The movements of the limbs were slow, just as that of the eyes, and just as the movements of the limbs are in paralysis agitans. The analogy suggests that such muscular rigidity is, in part at least, reflex (the afferent impression probably proceeding from the muscles themselves), and that there is a separation of the reflex centre from the voluntary centre, or at least a resistance to the action of the latter, which is only slowly overcome. 